\title{
VIOLENCIA OBSTÉTRICA: APROXIMACIÓN AL CONCEPTO Y DEBATE EN RELACIÓN A LA TERMINOLOGÍA EMPLEADA $^{1}$
}

\author{
Josefina Goberna-Tricas ${ }^{1}$ \\ Correo electrónico: jgoberna@ub.edu
}

1. Departamento de Enfermería de Salud Pública, Salud Mental y Maternoinfantil. Facultad de Medicina y Ciencias de la Salud. Universitat de Barcelona. ADHUC. Centro de Investigación en Teoría, Género y Sexualidad. Universitat de Barcelona.

Recibido: 10/05/2019 Aceptado: 25/06/2019

(c) (i) ()

\footnotetext{
${ }^{1}$ Este trabajo fue presentado en forma de ponencia en el III Congreso Internacional género, ética y cuidado, celebrado en Barcelona en mayo de 2018 y contribuye al Grupo de Investigación «Creación y Pensamiento de las mujeres» y al proyecto 2016ACUP083 financiado por RecerCaixa.
} 


\section{RESUMEN}

\section{Introducción}

Existe preocupación relacionada con el maltrato, la falta de respeto o la violencia que las instituciones sanitarias ejercen sobre las mujeres en la atención al parto. No existe unanimidad sobre la terminología adecuada para darle nombre. Este trabajo pretende profundizar en el significado de los diferentes términos usados para definir esta situación mediante una aproximación al concepto.

\section{Metodología}

Revisión de la literatura científica. Las palabras clave han sido: «Disrespect», «Abuse»; «Deshumanized care o obstetric violence», «childbirth» y «birth» y en español «abuso», «maltrato», «violencia obstétrica», «violencia institucional» o las acepciones «parto humanizado» 0 «parto respetado».

\section{Resultados y Discusión}

Bohren et al afirman que «Mistreatment» es un término más inclusivo que «obstetric violence», «dehumanized care» and «disrespect and abuse», puesto que engloba las experiencias de las mujeres y de los profesionales de la salud. Sin embargo, Jewkes y Penn-Kekana afirman que las definiciones de estos conceptos pueden ser demasiado amplias para la operacionalización. Lukasse et al. usan el término «abuse» y entienden que el concepto engloba «negligencias, abuso verbal, físico y sexual». Sadler establece una clara vinculación de la violencia obstétrica con la violencia de género y busca sus orígenes desde las raíces del nacimiento de la especialidad médica de la obstétrica.

\section{Conclusiones}

El concepto «violencia obstétrica» constituye una cuestión política de relaciones de poder que pretenden obediencia y sumisión de los cuerpos femeninos y da cuenta de que la experiencia de la maternidad se inscribe en el dominio de la biopolítica. Para que los profesionales de la salud acepten el término es importante reconocer que las prácticas sanitarias se desarrollan en un medio social en el que se ha ido construyendo un entramado histórico con un conjunto de creencias internas, reglas y prácticas, que reproducen las ideologías de género en las profesiones de la salud.

Palabras clave: violencia obstétrica; maltrato; falta de respeto; maternidad; parto; bioética. 


\section{ABSTRACT}

\section{Introduction}

There is concern related to the mistreatment, lack of respect or violence that health institutions exert on women in childbirth care. There is no unanimity on the proper terminology to name it. This work aims to explore the meaning of the different terms used to define this situation.

\section{Methodology}

Review of the scientific literature. The keywords were: «disrespect», «abuse»; «dehumanized care or obstetric violence», «childbirth» and «birth»; and in Spanish: «abuso», «maltrato», «violencia obstrétrica», «violencia institucional», and «parto humanizado» or «parto respetado».

\section{Results and Discussion}

Bohren et al state that «mistreatment» is a more inclusive term than «obstetric violence», «dehumanized care» and «disrespect and abuse», since it encompasses the experiences of women and health professionals. However, Jewkes and Penn-Kekana claim that the definitions of these concepts may be too broad to be operational. Lukasse et al. use the term «abuse» and understand that the concept encompasses «negligence, verbal, physical and sexual abuse». Sadler establishes a clear link between obstetric violence and gender violence and looks for its origins in the birth of obstetrics as a discipline.

\section{Conclusions}

The concept of «obstetric violence» is closely related to politics and power relations that seek obedience and submission of the female body while it also reveals that the experience of motherhood is part of the domain of biopolitics. For health professionals to accept the term, it is important to recognize that health practices are developed in a social environment in which a historical framework has been built with a set of internal beliefs, rules and practices that reproduce gender ideologies in health workers.

Keywords: obstetric violence; abuse; disrespect; maternity; birth; bioethics. 


\section{INTRODUCCIÓN}

En las últimas décadas se ha instaurado una preocupación, a nivel mundial, relacionada con el maltrato, la falta de respeto o la violencia que las instituciones sanitarias ejercen sobre las mujeres en el momento del parto hasta el punto de que la Organización Mundial de la Salud en 2015 anunciaba en su documento «Prevención y erradicación de la falta de respeto y el maltrato durante la atención del parto en centros de salud» que

todas las mujeres tienen derecho a recibir el más alto nivel de cuidados en salud, que incluye el derecho a una atención digna y respetuosa en el embarazo y en el parto, y el derecho a no sufrir violencia ni discriminación. El maltrato, la negligencia o la falta de respeto en el parto pueden constituirse en una violación de los derechos humanos fundamentales de las mujeres, descritos en las normas y los principios internacionales de derechos humanos. ${ }^{2}$

En España, fue en 2006 cuando se hizo visible un movimiento de demanda de cambios en la atención sanitaria al parto. El informe del Defensor del Pueblo se hizo eco de la petición de un significativo número de personas que solicitaban mejoras en los modelos de atención al proceso del parto, aconsejando la implementación de un protocolo para la atención de partos naturales no medicalizados al que se pudiesen acoger las mujeres/parejas que lo desearan. En esta misma línea de trabajo, el Observatorio de Salud de la Mujer del Ministerio de Sanidad y Consumo (2008) trató la atención al nacimiento en el II Foro de Mujeres, Salud y Género, y, en el tercero, abordó los indicadores sanitarios referentes al embarazo, parto y puerperio. Sin embargo, aunque la preocupación es ampliamente compartida por diferentes instituciones, investigadores y académicos, no existe unanimidad sobre la terminología adecuada para dar nombre a esta situación de falta de atención sanitaria adecuada, satisfactoria y acorde con el respeto a la dignidad de las mujeres durante el proceso del parto.

El lenguaje por medio de símbolos y significados llega a transformar la conciencia humana ocasionando nuevas formas de pensamiento y consecuentemente cambios en el imaginario social. Por ello, consideramos importante profundizar en el significado de los

\footnotetext{
${ }^{2}$ La cursiva es de la autora de este artículo.
} 
diferentes términos usados para definir esta situación a la vez que profundizamos en una aproximación al concepto.

\section{METODOLOGÍA}

Para este trabajo se ha realizado una revisión de la literatura científica accediendo a través de las bases de datos bibliográficas PubMed, Cuiden y Cuidatge. Las palabras clave usadas han sido: «disrespect», «abuse»; «dehumanized care» o «obstetric violence», «childbirth»y «birth», combinados con el booleano and y en español «abuso», «maltrato», «violencia obstétrica», «violencia institucional» o las acepciones «parto humanizado» o «parto respetado» para referirse a modelos asistenciales que quieren poner fin a esta situación. Tras una primera búsqueda y la lectura de los resúmenes, se seleccionaron solamente aquellos trabajos que desarrollaban teóricamente los conceptos.

\section{RESULTADOS Y DISCUSIÓN}

Bohren et al en una revisión de 2015 afirman que «mistreatment» es un término más inclusivo que «obstetric violence», «dehumanized care», «disrespect» $\mathrm{y}$ «abuse», puesto que engloba de mejor forma las experiencias de las mujeres y de los profesionales de la salud; tanto aquellas experiencias activas (por ejemplo si existe abuso de forma intencionada) como aquellas que se producen de forma pasiva (cuando por ejemplo se produce alguna negligencia en la atención, a veces debida a déficits estructurales); tanto las relacionadas con los comportamientos individuales (abusos verbales) como las relacionadas con las condiciones de las instalaciones sanitarias (falta de camas, falta de condiciones de privacidad...). La Organización Mundial de la Salud (2015) usa también el término «mistreatment» $\mathrm{y}$ recomienda desarrollar instrumentos para medirlo $\mathrm{y}$ evaluarlo. Sin embargo Jewkes y Penn-Kekana (2015) afirman que las definiciones que los autores realizan de estos conceptos pueden ser demasiado amplias para la operacionalización y aconsejan el desarrollo de una tipología del maltrato que se beneficiaría de un enfoque más específico sobre el uso intencional de la violencia y las deficiencias estructurales que equivalen a violencia.

Por su parte, Lukasse et al. (2015) usan el término «abuse» y entienden que el concepto engloba «negligencias, abuso verbal, físico y en ocasiones incluso sexual». Para 
el uso de este término se basan en la definición de Bruggemann et al. (2012), quienes definen el «abuso en atención sanitaria» como:

Experiencia subjetiva de los pacientes en sus encuentros con el sistema de salud, caracterizado por situaciones desprovistas de cuidado, en las que los pacientes sufren y sienten que pierden su valor como seres humanos.

Estos autores entienden la experiencia de abuso como un componente subjetivo relacionado con las expectativas previas de los pacientes. Del mismo modo, presuponen que las mujeres, durante el proceso del parto, ocupan el rol de pacientes en el sistema sanitario, aunque reconocen que las mujeres durante el parto pueden ser más vulnerables a padecer esta percepción de abuso o de maltrato debido a las altas expectativas previas. Por último, afirman que las mujeres pueden padecer síntomas de estrés postraumático tras estas experiencias negativas, manifestando problemas de trastornos del sueño, estrés y pobre percepción de su estado de salud.

Es en el contexto de Latinoamérica y el Caribe donde surgen los términos de «violencia obstétrica», «cuidados deshumanizados»o «discriminación», equiparándose la violencia obstétrica a otros tipos de violencia hacia las mujeres. Los estudios suelen focalizarse hacia prácticas clínicas inadecuadas y tendentes a contemplar embarazo y parto como situaciones patológicas en que las instituciones sanitarias toman el poder adoptando una actitud paternalista e incluso patriarcal frente a las mujeres gestantes. Se incluyen como prácticas de violencia obstétrica: cesáreas, episiotomías innecesarias o esterilizaciones no consentidas.

Algunas de las investigaciones relacionadas con el término «violencia obstétrica» se refieren explícitamente a la violencia estructural e institucional del sistema sanitario que refleja las relaciones de poder y la falta de equidad vinculada a cuestiones de género presentes en los servicios y programas de salud reproductiva (Castro, 2019; Goer, 2010). La consideración de la violencia obstétrica como violencia de género ha permitido tipificarla en los ordenamientos jurídicos de estos países. Así, Venezuela define la violencia obstétrica —-mediante la Ley orgánica sobre el derecho de las mujeres a una vida libre de violencia - de la siguiente forma:

Violencia obstétrica: Es la apropiación del cuerpo y procesos reproductivos de las mujeres por personal de salud, que se expresa en 
un trato deshumanizador, en un abuso de medicalización y patologización de los procesos naturales, trayendo consigo pérdida de autonomía y capacidad de decidir libremente sobre sus cuerpos y sexualidad, impactando negativamente en la calidad de vida de las mujeres.

Posteriormente, algunos estados de México en 2008 y Argentina en 2009 (Belly, 2013) integraron también en su marco legal dicha definición. En el caso de Argentina, $L a$ Ley de Protección Integral para prevenir, erradicar y sancionar la violencia contra las mujeres la define como:

Aquella que ejerce el personal de salud sobre el cuerpo y los procesos reproductivos de las mujeres, expresada en un trato deshumanizado, un abuso de medicalización y patologización de los procesos naturales.

Chile y Costa Rica han introducido también relevante literatura con el uso de este término, aunque todavía no han legislado en este sentido. Brasil y Argentina han pedido en su legislación «La humanización del nacimiento».

Savage y Castro en un reciente estudio de 2017 reconocen que algunos autores usan los diferentes términos enumerados de forma indistinta, pero un análisis más pormenorizado del uso de dichos conceptos reconoce que el uso del término «violencia obstétrica en latinoamerica» suele usarse para referirse a discriminación hacia ciertos colectivos de mujeres, especialmente indígenas o afrodescendientes, y a la excesiva medicalización de los procesos fisiológicos enfatizando el elevado número de cesáreas o episiotomías no justificadas, poniendo también énfasis en las relaciones asimétricas de poder que se establecen entre usuarias y profesionales (especialmente con profesionales de la medicina). En este sentido, también Sadler et al. (2016) enfatizan que, aunque los términos «disrespect», «abuse» $\mathrm{y}$ «mistreatment» durante el parto son usados como sinónimos por muchos investigadores, «violencia obstétrica» se muestra como el más adecuado. Sadler establece una clara vinculación de la violencia obstétrica con la violencia de género. La definición de violencia obstétrica, además de reconocer el tratamiento deshumanizado, destaca fundamentalmente su dimensión exclusivamente obstétrica, diferenciada de las situaciones de falta de respeto en otros ámbitos sanitarios y busca sus orígenes en el largo periodo histórico desarrollado desde la expropiación del saber femenino por la práctica médica en el siglo XVII hasta el nacimiento de la 
especialidad médica de la obstétrica en el siglo XIX (Goberna, 2016). Silvia Federici (2010), usando los constructos teóricos ofrecidos por Foucault y tensionándolos en un constante proceso crítico, describe como durante la transición del feudalismo al capitalismo, las mujeres fueron desterradas de sus propios cuerpos de forma paulatina, fundamentalmente en lo que a la sexualidad y la reproducción se refiere. Belly (2013) sitúa la experiencia de la maternidad en el espacio de dominio de la biopolítica afirmando que «la negación del beneficio de prácticas y saberes tradicionales relativos al parto coloca a los profesionales de la salud cómo los únicos autorizados a intervenir en el cuerpo de las mujeres». A su vez, Sadler visibiliza el componente estructural de la violencia obstétrica y la distingue de otras formas de violencia que tienen lugar en el ámbito hospitalario, situándola como una cuestión feminista, un caso de violencia de género, puesto que las mujeres, aunque estén de parto, siguen siendo personas sanas y embarazo y parto no constituyen enfermedades, sino que deberían conceptualizarse como eventos sexuales. Así, la violencia obstétrica, según estos autores, puede ser interpretada como una violación.

Una de las razones por las cuales el término violencia obstétrica no está más extendida es que los profesionales de la salud se resisten al uso del concepto de violencia, que es contrario a su ethos. Como Diniz y Oliveira (1998) explican, ello hizo que la Red para la Humanización del Parto y Nacimiento en Brasil decidiera no hablar abiertamente sobre violencia durante los años noventa del siglo XX, favoreciendo expresiones como «humanizar el parto». No obstante, los mismos organizadores que acuñaron el término «humanización del parto» reconocen que los significativos cambios sociales que han ocurrido desde entonces han llevado a que el debate surgido con el uso de estos términos comenzara a ser conceptualizado como una cuestión de violencia de género y de derechos humanos.

Las estructuras de poder incrustadas y reproducidas en biomedicina construyen un currículum oculto en la formación de los profesionales de la salud que favorece su socialización en la aceptación de normas, disciplina corporativa y castigo, mientras que los aspectos emocionales y las dimensiones de la atención se descuidan (Goberna y Boladeras, 2018). Las condiciones de trabajo de muchos profesionales de la salud también deben visibilizarse como formas de falta de respeto y abuso. El uso del concepto violencia obstétrica contribuye a la visibilización de esta violencia institucional y evitan «culpar a los trabajadores de la salud como un grupo, cuando se trata del resultado de una situación 
estructural de la cual los profesionales son también víctimas» (Jewkes y Penn-Kekana, 2015). La evidencia científica muestra que el personal de salud expuesto a la violencia que se ejerce en los paritorios puede sufrir estrés traumático o fatiga por compasión, entendida como una exposición traumática secundaria a factores estresantes en el seno de su propia organización, similares a los experimentados por las propias mujeres gestantes (Olza, 2014).

\section{CONCLUSIONES}

El concepto «violencia obstétrica» entendido como cuestión política de relaciones de poder que pretenden la obediencia y sumisión de los cuerpos femeninos nos permite dar cuenta de que la experiencia de la maternidad conforma uno de los espacios de dominio de la biopolítica que coloca a los profesionales de la salud como los únicos autorizados a intervenir en el cuerpo de las mujeres desplazando a las mujeres del rol protagonista en su propio embarazo y parto.

Nombrar en voz alta la violencia obstétrica ha permitido colocar el problema del maltrato y la falta de respeto en atención al parto en las agendas de política pública y feminista, evitando centrar el debate única y exclusivamente en negligencias individuales que tienen el potencial de generar hostilidad improductiva en el debate de falta de respeto y abuso en el parto, especialmente entre los profesionales de la salud. Para que los profesionales de la salud acepten el uso del término es importante reconocer que la biomedicina y las prácticas médicas y sanitarias se desarrollan en un medio social y en un sistema cultural en el que se ha ido construyendo un entramado histórico complejo con un conjunto coherente de creencias internas, reglas y prácticas que responden y reproducen las ideologías de género en las profesiones de la salud.

Es necesario un análisis más amplio, centrado en las dimensiones culturales y sociales en que se incrusta el fenómeno de la violencia obstétrica, lo que puede permitir un cambio de mirada que deje de limitarse a las víctimas (mujeres) y victimarios (profesionales de la salud) para ampliar la mirada hacia el reconocimiento de la socialización ubicua de hombres y mujeres en formas naturalizadas, y por lo tanto invisibles, de violencia y dinámicas de poder entre grupos. 


\section{BIBLIOGRAFÍA}

BELLI, L. La violencia obstétrica: otra forma de violación a los derechos humanos. En: Revista Redbioética/UNESCO. 2013, vol. 1, núm. 7, pp. 25-34.

BoHren, M. A., et al. The mistreatment of women during childbirth in health facilities globally: a mixed-methods systematic review. En: PLoSMed. 2015, vol. 12, núm. 6, pp. 32.

Brüggemann, A. J.; WiJMA, B.; Swahnberg, K. Abuse in health care: a concept analysis. In: Scandinavian Journal of Caring Sciences. 2012, vol. 26, pp. 123-132.

Cámara de Diputados del H. Congreso de la Unión. Ley General de Acceso de las Mujeres a una vida Libre de Violencia. México: 13 de abril de 2018 (última reforma publicada). Disponible en:

<http://www.diputados.gob.mx/LeyesBiblio/pdf/LGAMVLV_130418.pdf>.

CAstro, A. Witnessing Obstetric Violence during Fieldwork: Notes from Latin America.

In: Health and Human Rights Journal. 2019. Disponible en: <www.hhrjournal.org/2019/06/witnessing-obstetric-violence-during-fieldworknotes-from-latin-america/>.

DEFENSOR DEL PUEBLO ESPAÑA. Informe anual 2006. Madrid: Oficina del Defensor del Pueblo, 2007.

Diniz, S. G.; D'OliveIRA, A. F. Gender violence and reproductive health. In: International Journal of Gynecology \& Obstetrics. 1998, vol. 63 Suppl. 1, pp. 33-42.

FEDERICI, S. Calibán y la bruja. Mujeres, cuerpo y acumulación originaria. Madrid: Traficantes de Sueños, 2010.

GOBERNA TRICAS, J. El nacimiento de la burguesía y la pérdida de poder de las matronas. En: Boladeras Cucurella, Margarita; Goberna Tricas, Josefina (eds.). Bioética de la maternidad. Barcelona: Universidad de Barcelona, 2016, pp. 41-53.

GOBERnA TRICAS, J.; BOLADERAS, M. Análisis del concepto de violencia obstétrica desde las perspectivas legal, médica, filosófica, sociopolítica y pedagógica. En: GobernaTricas, J.; Boladeras, M. (eds.). El concepto violencia obstétrica y el debate actual en atención al nacimiento. Madrid: Tecnos, 2018, pp. 39-68.

GOBIERNO DE VENEZUELA. Ley orgánica sobre el derecho de las mujeres a una vida libre de violencia de Venezuela. Artículo 15, párrafo 13. Venezuela: 2017. Disponible en: <www.bdigital.unal.edu.co/48830/1/leyorganicasobreelderecho.pdf〉. 
GOER, H. Cruelty in maternity wards: Fifty years later. In: Journal of Perinatal Education. 2010, vol. 19, núm. 3, pp. 33-42.

Jewkes, R; Penn-KeKanA, L. Mistreatment of Women in Childbirth: Time for Action on This Important Dimension of Violence against Women. En: PLoS Med. 2015, vol. 12, núm. 6.

LUKASSE M., et al. Prevalence of experienced abuse in healthcare and associated obstetric characteristics in sixEuropean countries. In: Acta Obstet Gynecol Scand. 2015, vol. 94, pp. 508-517.

Ministerio de SANIDAd y POLÍTICA SOcial. Guía de Práctica clínica sobre la atención al parto normal en el Sistema Nacional de Salud. Vitoria-Gasteiz: Servicio Central de Publicaciones del Gobierno Vasco, 2010.

ObServatorio de SAlud de la Mujer y DEl Sistema Nacional De Salud. Estrategia de Atención al Parto Normal. Madrid: Ministerio de Sanidad y Consumo, 2007.

OLZA FERNÁNDEZ, I. Estrés postraumático secundario en profesionales de la atención al parto. Aproximación al concepto de violencia obstétrica. En: Cuadernos de medicina psicosomática y psiquiatria de enlace. 2014, vol. 111, pp. 79-83

ORganización Mundial DE LA SALUd (OMS). Prevención y erradicación de la falta de respeto y el maltrato durante la atención del parto en centros de salud. Ginebra: OMS, 2014. Disponible en:

<www.who.int/reproductivehealth/topics/maternal_perinatal/statement-childbirth/es/>.

SADLER, M., et al. Moving beyond disrespect and abuse: addressing the structural dimensions of obstetric violence. In: Reproductive Health Matters. 2016, vol. 24, núm. 47, pp. 47-55.

SAVAge, V.; CAstro, A. Measuring mistreatment of women during childbirth: a review of terminology and methodological approaches. In: Reprod Health. 2017, vol. 14, núm. 1, p. 138. 